\title{
Evaluating the effect of delayed activation of rapid response teams on patient outcomes: a systematic review protocol
}

\author{
Michael K. Xu*, Kathleen G. Dobson², Lehana Thabane ${ }^{1}$ and Alison E. Fox-Robichaud²]
}

\begin{abstract}
Background: Rapid response teams have been widely adopted across the world. Although evidence for their efficacy is not clear, they remain a popular means to detect and react to patient deterioration. This may in part be due to there being no standardized approach to their usage or implementation. A key component of their ability to be effective is the speed of response.
\end{abstract}

Objective: The objective of this review is to evaluate the effect of delayed response by rapid response teams on hospital mortality (primary), cardiac arrest, and intensive care transfer rates (secondary).

Methods: This review will include randomized and non-randomized studies which examined the effect of delayed response times by rapid response teams on patient mortality, cardiac arrest, and intensive care unit admission rates. This review will include studies of adult patients who have experienced a rapid response team consultation. The search strategy will utilize a combination of keywords and MeSH terms. MEDLINE and Embase will be searched, as well as examining gray literature. Two reviewers will independently screen retrieved citations to determine if they meet inclusion criteria. Studies will be selected that provide information about the impact of response time on patient outcomes.

Comparisons will be made between consults that arrive in a timely manner and consults that are delayed. Quality assessment of randomized studies will be conducted in accordance with guidelines from the Cochrane Handbook for Systematic Reviews of Interventions. Quality assessment of non-randomized studies will be based on the Risk of Bias in Non-randomized Studies-of Interventions (ROBINS-I) assessment tool. Results of the review will be reported according to the Preferred Reporting Items for Systematic Reviews and Meta-Analyses guidelines.

Discussion: This systematic review will identify and synthesize evidence around the impact of delayed response by rapid response teams on patient mortality, cardiac arrest, and intensive care transfer rates.

Systematic review registration: PROSPERO Registration: CRD42017071842.

Keywords: Systematic review, Rapid response team, Critical care, Medical emergency team, Intensive care, Mortality, Delay

\footnotetext{
* Correspondence: xum5@mcmaster.ca

${ }^{1}$ Department of Health Research Methods, Evidence, and Impact, McMaster

University, DBRI C5-106, 237 Barton St. East, Hamilton, ON L8L 2X2, Canada

Full list of author information is available at the end of the article
} 


\section{Background}

Patients exhibit physiological deterioration prior to cardiac arrest [1-4]. Rapid response systems are designed to detect this physiological deterioration and activate a critical care response to the bedside to assess and intervene $[5,6]$. Rapid response systems operate with an afferent arm, an early warning score or trigger mechanism and an efferent arm, a rapid response team. In the context of this review, rapid response teams are defined as critical care physician-led teams designed to provide rapid critical care for in-patients on general hospital wards. In recent years, rapid response systems have been adopted globally with multiple nations mandating their use in major hospitals [7-10].

Current evidence is mixed as to the effectiveness of rapid response systems at the reduction of patient mortality, with most evidence suggesting some effect at reducing cardiac arrest rates [4,11-13]. Several single-center studies as well as a meta-analysis have found improved outcomes with rapid response system implementation [12, 14-16]. However, another meta-analysis and the only multicenter randomized control trial to date have not found strong evidence to support the effectiveness of rapid response systems $[2,3]$. In many of these studies, the quality of the rapid response system as a systematic intervention itself has not been evaluated [17-20]. Previous reviews of rapid response systems have treated rapid response systems as if they were of equal quality and had comparable operating procedures [2, 17, 19, 21]. Few studies have reported on the response times of their efferent arms and how this may impact patient outcomes [13, 22, 23].

The timely identification and response to critical deterioration in patients is key to the effectiveness of a rapid response system at decreasing patient mortality, ICU admissions, and cardiac arrest rates [24]. Some studies have suggested that a delay between identification of deterioration and the rapid response team arriving is associated with a higher mortality [25, 26]. In addition, there currently exists no standardized guideline as to what constitutes a delayed activation of the rapid response system.

\section{Objectives}

The primary objective of this systematic review is to identify and critically assess the existing literature assessing the effect of delayed activation of rapid response teams on hospital mortality among in-hospital patients. The secondary objective is to assess the effect of delayed activation of rapid response teams on cardiac arrests and ICU transfers. This will be conducted by examining the association between increased response times and mortality, ICU transfers, or cardiac arrest.

The secondary objective is to evaluate these studies for what they define as a delayed activation of a rapid response team, how rapid response teams are triggered, and if identifiable, where potential delays may occur in the activation process. The review will be reported according to the Preferred Report Items for Systematic Reviews and Meta-Analyses (PRISMA) guidelines [27].

\section{Methods}

This systematic review protocol has been designed with the PRISMA-P guidelines for reporting systematic reviews in mind [27]. A checklist of PRISMA-P criteria met is included in Additional file 1. This protocol has been registered with the PROSPERO International Prospective Register of Systematic Reviews (PROSPERO CRD42017071842).

\section{Eligibility criteria}

Included studies examined populations of hospitalized adult ( $\geq 18$ years) patients that experienced a rapid response or medical emergency team call.

Studies assessing the effect of delayed activation, or response teams of rapid response teams or medical emergency teams will be considered for inclusion. These studies will be included if there are clear outlined criteria for what calling criteria would be for the activation of these teams, without any limitation on the afferent or triggering system. Studies must give reference to what constitutes a delayed or early call, or examine the relationship between response time and patient outcomes for inclusion. Studies must include a control group. Outcomes of interest are defined as of the following critical events: patient mortality, cardiac arrest, and ICU admission. These outcomes were selected as they are the outcomes for which rapid response teams seek to prevent and have been assessed against in previous studies $[3,18,28]$. There is no minimum number or percentage of patients that experience these outcomes needed for inclusion in this review. No exclusions will be placed on country; studies must be published in English.

Studies will be excluded if they meet any of the following criteria: do not report on patient outcomes following the arrival of a rapid response team, do not describe the criteria or methods for the activation of a rapid response team, do not report on quantitative data regarding the delayed activation (i.e., measures of association) or the length of delay, and/or are editorials or commentaries.

\section{Search strategy}

The search strategy aims to find both published literature and any potential gray literature between January 1, 1990, and the time of the start of the review process. A three-step strategy will be utilized in this review. Initially, a limited search of the MEDLINE database will be undertaken to determine keywords of interest that may be used in the title, abstract, and indexing of relevant 
literature. A draft of the search strategy for MEDLINE can be found in Additional file 2. Following this, a second search using keywords identified previously will be undertaken across MEDLINE, EMBASE, Cochrane, and CINAHL. Additionally, full reference lists of included studies will be screened. A PRISMA flowchart will illustrate the study selection process and reasons for exclusion. Studies will be assessed for eligibility by one reviewer and checked by a second.

\section{Data abstraction}

Data abstraction will be conducted independently by two reviewers (MX, KD). Data extracted will be entered into a spreadsheet. The following data items will be abstracted when available: (i) study identification items (first author, year of publication), (ii) study design characteristics (intervention, calling criteria for rapid response team, sample size, control group, defined time for delayed activation, duration of data collection), (iii) target population, (iv) setting (nationality, healthcare environment, maturity of response team), and (v) clinical outcomes (cardiac arrest, ICU admission, mortality).

\section{Risk of bias/quality assessment}

In order to assess the quality of research, two independent reviewers (MX, KD) will assess the risk of bias using the Cochrane Collaboration's tool for assessing risk of bias in RCTs, as well as the ROBINS-I assessment tool for nonRCT studies $[29,30]$. Each study will be assessed for procedures specified in their respective appropriate tool. Studies will be rated as showing a "low," "moderate," or "high" risk of bias according to criteria specified in each tool.

\section{Data synthesis}

Given the anticipated paucity of literature, published or otherwise on this topic, this systematic review is intended to be exploratory, inclusive, and descriptive in nature. The primary objective of this review is to identify and appraise literature regarding the delayed activation of rapid response systems. However, a meta-analysis may be considered dependent on the body of literature identified.

\section{Discussion}

This systematic review will add to previous research on rapid response systems by synthesizing, summarizing, and discussing the existing literature on the effect that delayed activation of the rapid response team has on patient outcomes. To the authors' knowledge, this is the first systematic review to specifically examine the impact that delayed activation has. Prior systematic reviews have evaluated rapid response systems or rapid response teams as interventions, but none have evaluated the effect of quality of these systems as an intervention and how degradation of their effectiveness impacts patient outcomes. The proposed review will provide a valuable overview and synthesis of a potential area for improvement and discussion regarding rapid response systems and their use.

The proposed review will go beyond summarizing the existing evidence, by also looking at factors listed contributing to delays in activation. In this way, areas needing further study can be identified and potential poor practices in the deployment of these systems can be highlighted.

Rapid response systems possess high face validity for being an effective systematic intervention for the early detection and management of critical deterioration in patients; however, the literature has provided mixed evidence for this effect. Given that these systems rely on rapid response, it is surprising that there is little literature regarding how to best implement and use these systems, especially with respect to response times. With the rapid adoption of these systems, it is crucial to determine how increased response times may degrade the effectiveness of rapid response systems at improving patient outcomes. The proposed systematic review is urgently needed and will substantially add to the current evidence, helping to shape and guide future practice regarding rapid response systems.

\section{Additional file}

Additional file 1: PRISMA-P checklist. (DOCX $31 \mathrm{~kb}$ )

Additional file 2: Draft search strategy. (DOC $24 \mathrm{~kb}$ )

Acknowledgements

Not applicable.

Funding

Not applicable.

Availability of data and materials

The datasets used and/or analyzed during the current study are available from the corresponding author on reasonable request.

\section{Authors' contributions \\ $M X$ and AFR were involved in the concept and review design of the study plan. LT provided guidance regarding methods of data abstraction and quality assessment. KD reviewed the design of the study. All authors reviewed and revised the manuscript and read and approved the final manuscript. AFR is the guarantor of this work.}

Ethics approval and consent to participate

Not applicable.

Consent for publication

Not applicable.

Competing interests

The authors declare that they have no competing interests. 


\section{Publisher's Note}

Springer Nature remains neutral with regard to jurisdictional claims in published maps and institutional affiliations.

\section{Author details}

'Department of Health Research Methods, Evidence, and Impact, McMaster University, DBRI C5-106, 237 Barton St. East, Hamilton, ON L8L 2X2, Canada. ${ }^{2}$ Department of Medicine, McMaster University, Hamilton, Canada. ${ }^{3}$ Dalla Lana School of Public Health, University of Toronto, Toronto, Canada.

Received: 1 September 2017 Accepted: 23 February 2018 Published online: 09 March 2018

\section{References}

1. Buist MD, Jarmolowski E, Burton PR, Bernard SA, Waxman BP, Anderson J. Recognising clinical instability in hospital patients before cardiac arrest or unplanned admission to intensive care. A pilot study in a tertiary-care hospital. Med J Aust. 1999:171:22-5.

2. Chan PS, Jain R, Nallmothu BK, Berg RA, Sasson C. Rapid response teams: a systematic review and meta-analysis. Arch Intern Med. 2010;170:18-26.

3. Hillman K. Introduction of the medical emergency team (MET) system: a cluster-randomised controlled trial. Lancet. 2005:365:2091-7.

4. Cretikos M, Chen J, Hillman K, Bellomo R, Finfer S, Flabouris A. The objective medical emergency team activation criteria: a case-control study. Resuscitation. 2007;73:62-72.

5. Bellomo R. Rapid-response teams. NEnglJ Med. 2011;365(Table 2):139-46.

6. Jones D, Bellomo R. Introduction of a rapid response system: why we are glad we MET. Crit Care. 2006;10:121

7. Brunsveld-Reinders AH, Ludikhuize J, Dijkgraaf MGW, Arbous MS, de Jonge E. Unexpected versus all-cause mortality as the endpoint for investigating the effects of a rapid response system in hospitalized patients. Crit Care. 2016;20:1

8. Smith GB, Prytherch DR, Meredith P, Schmidt PE, Featherstone PI. The ability of the National Early Warning Score (NEWS) to discriminate patients at risk of early cardiac arrest, unanticipated intensive care unit admission, and death. Resuscitation. 2013;84:465-70.

9. Tanguay BT, Bartel R. Implementation of a unique RRT model in a tertiary care centre in Western Canada. Can J Crit Care Nurs. 2015;28(C):34-7.

10. Lobos A-T, Fernandes R, Willams K, Ramsay C, McNally JD. Routine medical emergency team assessments of patients discharged from the PICU: description of a medical emergency team follow-up program. Pediatr Crit Care Med A J Soc Crit Care Med World Fed Pediatr Intensive Crit Care Soc. 2015;16:359-65.

11. Winters BD, Weaver SJ, Pfoh ER, Yang T, Cuong J, Dy SM. Rapid-response systems as a patient safety strategy: a systematic review. Ann Intern Med. 2013;158(5 PART 2):417-25

12. Salvatierra G, Bindler RC, Corbett C, Roll J, Daratha KB. Rapid response team implementation and in-hospital mortality. Crit Care Med. 2014;42:2001-6.

13. Calzavacca P, Licari E, Tee A, Egi M, Downey A, Quach J, et al. The impact of rapid response system on delayed emergency team activation patient characteristics and outcomes-a follow-up study. Resuscitation. 2010;81:31-5.

14. De Jong A, Jung B, Daurat A, Chanques G, Mahul M, Monnin M, et al. Effect of rapid response systems on hospital mortality: a systematic review and meta-analysis. Intensive Care Med. 2016:42:615-7.

15. Gerdik C, Vallish RO, Miles K, Godwin SA, Wludyka PS, Panni MK. Successful implementation of a family and patient activated rapid response team in an adult level 1 trauma center. Resuscitation. 2010;81:1676-81.

16. Offner PJ, Heit J, Roberts R. Implementation of a rapid response team decreases cardiac arrest outside of the intensive care unit. J Trauma. 2007; 62:1223-8.

17. McNeill G, Bryden D. Do either early warning systems or emergency response teams improve hospital patient survival? A systematic review. Resuscitation. 2013:84:1652-67.

18. Ludikhuize J, Brunsveld-Reinders AH, Dijkgraaf MGW, Smorenburg SM, de Rooij SEJ a, Adams R, et al. Outcomes associated with the nationwide introduction of rapid response systems in The Netherlands. Crit Care Med. 2015:43:2544-51.

19. de Jong A, Jung B, Daurat A, Chanques G, Mahul M, Monnin M, et al. Effect of rapid response systems on hospital mortality: a systematic review and meta-analysis. Intensive Care Med. 2016;42:1-3.
20. Aitken LM, Chaboyer WR, Vaux AR, Crouch SR, Burmeister ER, Daly M, et al. Continuing professional development questions effect of a 2 tier rapid response system on patient outcome and staff satisfaction. Aust Crit Care. 2015:28:115

21. Tirkkonen J, Tamminen T, Skrifvars MB. Outcome of adult patients attended by rapid response teams: a systematic review of the literature. Resuscitation. 2017;112:43-52.

22. Boniatti MM, Azzolini N, Viana MV, Ribeiro BSP, Coelho RS, Castilho RK, et al. Delayed medical emergency team calls and associated outcomes. Crit Care Med. 2014;42:26-30.

23. Tirkkonen J, Ylä-Mattila J, Olkkola KT, Huhtala H, Tenhunen J, Hoppu S. Factors associated with delayed activation of medical emergency team and excess mortality: an Utstein-style analysis. Resuscitation. 2013;84:173-8.

24. Wallis C. Timing is everything. Bercut. 2015;26:1.

25. Chen J, Bellomo R, Flabouris A, Hillman K, Assareh H, Delayed Emergency OL. Team calls and associated hospital mortality: a multicenter study. Crit Care Med. 2015:43:2059-65.

26. Barwise A, Thongprayoon C, Gajic O, Jensen J, Herasevich V, Pickering BW. Delayed rapid response team activation is associated with increased hospital mortality, morbidity, and length of stay in a tertiary care institution. Crit Care Med. 2016:44:54-63.

27. Moher D, Shamseer L, Clarke M, Ghersi D, Liberati A, Petticrew M, et al. Preferred reporting items for systematic review and meta-analysis protocols (PRISMA-P) 2015 statement. Syst Rev. 2015:4:1.

28. Jones D, George C, Hart GK, Bellomo R, Martin J. Introduction of medical emergency teams in Australia and New Zealand: a multi-centre study. Crit Care. 2008;12:R46

29. Higgins, Altman, Gotzsche, Juni, Moher, Oxman, et al. The Cochrane Collaboration's tool for assessing risk of bias in randomised trials. BMJ. 2011; d5928:343.

30. Sterne JA, Hernán MA, Reeves BC, Savović J, Berkman ND, Viswanathan M, et al. ROBINS-I: a tool for assessing risk of bias in non-randomised studies of interventions. BMJ. 2016;i4919:355.

\section{Submit your next manuscript to BioMed Central and we will help you at every step:}

- We accept pre-submission inquiries

- Our selector tool helps you to find the most relevant journal

- We provide round the clock customer support

- Convenient online submission

- Thorough peer review

- Inclusion in PubMed and all major indexing services

- Maximum visibility for your research

Submit your manuscript at www.biomedcentral.com/submit
Biomed Central 